\title{
Double Beta Decay Search with CUPID-0: Results and Perspectives
}

\author{
L. Cardani ${ }^{* a}$, O. Azzolini ${ }^{b}$, M. T. Barrera ${ }^{b}$, J. W. Beeman ${ }^{c}$, F. Bellini ${ }^{a, d}$, M. Beretta ${ }^{e, f}$, \\ M. Biassoni ${ }^{f}$, C. Brofferio ${ }^{e, f}$,C. Bucci ${ }^{g}$, L. Canonica ${ }^{g}$, S. Capellie ${ }^{e, f}$, P. Carniti ${ }^{e, f}$, \\ N. Casali ${ }^{a}$, L. Cassina ${ }^{e, f}$, M. Clemenza ${ }^{e, f}$, O. Cremonesi $^{f}$, A. Cruciani $^{a}$, \\ A. D’Addabbo ${ }^{g, h}$, I. Dafinei ${ }^{a}$, S. Di Domizio ${ }^{i, l}$, F. Ferroni ${ }^{a, d}$, L. Gironi ${ }^{e, f}$, A. Giuliani ${ }^{m, n}$, \\ P. Gorla ${ }^{g}$, C. Gotti ${ }^{e, f}$, G. Keppel ${ }^{b}$, M. Martinez ${ }^{a, d}$, S. Morganti ${ }^{a}$, S. Nagorny ${ }^{g, h}$, \\ M. Nastasi ${ }^{e, f}$, S. Nisi ${ }^{g}$, C. Nones ${ }^{o}$, D. Orlandi ${ }^{g}$, L. Pagnanini ${ }^{e, f}$, M. Pallavicini ${ }^{i, l}$, \\ V. Palmieri ${ }^{b}$, L. Pattavina ${ }^{g, h}$, M. Pavan ${ }^{e, f}$, G. Pessina ${ }^{f}$, V. Pettinacci ${ }^{a}$, S. Pirro ${ }^{g}$, \\ S. Pozzi ${ }^{e, f}$, E. Previtali $f$, A. Puiuu ${ }^{e, f}$, C. Rusconi ${ }^{g, p}$, K. Schäffner ${ }^{g, h}$, C. Tomei ${ }^{a}$, \\ M. Vignati ${ }^{a}$ and A. Zolotarova ${ }^{o}$ \\ ${ }^{a}$ INFN - Sezione di Roma, Roma I-00185 - Italy, \\ ${ }^{b}$ INFN - Laboratori Nazionali di Legnaro, Legnaro (Padova) I-35020 - Italy, \\ ${ }^{c}$ Materials Science Division, Lawrence Berkeley National Laboratory, Berkeley, CA 94720 - \\ USA, \\ ${ }^{d}$ Dipartimento di Fisica, Sapienza Università di Roma, Roma I-00185 - Italy, \\ ${ }^{e}$ Dipartimento di Fisica, Università di Milano-Bicocca, Milano I-20126 - Italy, \\ ${ }^{f}$ INFN - Sezione di Milano Bicocca, Milano I-20126 - Italy, \\ ${ }^{g}$ INFN - Laboratori Nazionali del Gran Sasso, Assergi (L'Aquila) I-67010 - Italy, \\ ${ }^{h}$ Gran Sasso Science Institute, 67100, L’Aquila - Italy, \\ ${ }^{i}$ Dipartimento di Fisica, Università di Genova, Genova I-16146 - Italy, \\ ${ }^{l}$ INFN - Sezione di Genova, Genova I-16146 - Italy, \\ ${ }^{m}$ CSNSM, Univ. Paris-Sud, CNRS/IN2P3, Université Paris-Saclay, 91405 Orsay, France, \\ ${ }^{n}$ DiSAT, Università dell'Insubria, 22100 Como, Italy, \\ ${ }^{o}$ IRFU, CEA, Université Paris-Saclay, F-91191 Gif-sur-Yvette, France, \\ ${ }^{p}$ Department of Physics and Astronomy, University of South Carolina, Columbia, SC 29208 - \\ USA. \\ E-mail: laura.cardanieromal.infn.it.
}

CUPID-0 is the first large mass experiment based on cryogenic calorimeters (bolometers) that implements the dual read-out of light and heat for background rejection. The detector, consisting of 24 enriched $\mathrm{Zn}^{82} \mathrm{Se}$ crystals $\left(5.28 \mathrm{~kg}\right.$ of ${ }^{82} \mathrm{Se}$ ), is taking data in the underground LNGS (Italy) from March 2017. In this contribution we present the analysis that allowed to set the most stringent limit on the half-life of neutrino-less double beta decay of ${ }^{82} \mathrm{Se}$. We prove that the particle identification, enabled by the simultaneous read-out of heat and light, provides an unprecedented background level for cryogenic calorimeters of few $10^{-3}$ counts $/ \mathrm{keV} / \mathrm{kg} / \mathrm{y}$. Finally, we discuss the impact of these results on next generation projects.

EPS-HEP 2017, European Physical Society conference on High Energy Physics

5-12 July 2017

Venice, Italy

* Speaker. 


\section{Introduction}

Several extensions of the Standard Model of Particle Physics predict that double beta decay $(\beta \beta)$ could occur also with no-neutrino emission [1]. The observation of this decay $(0 v \beta \beta)$ would give precious insights in the Physics beyond the Standard Model, proving the existence of a process that violates the conservation of the total lepton number; furthermore, it would allow to establish the fundamental nature of neutrinos, as this decay can occur only if neutrinos, in contrast to all the other known fermions, are Majorana particles, i.e. they coincide with anti-neutrinos [2].

Only certain nuclei are of interest for $0 v \beta \beta$, such as ${ }^{76} \mathrm{Ge},{ }^{82} \mathrm{Se},{ }^{100} \mathrm{Mo},{ }^{136} \mathrm{Xe},{ }^{130} \mathrm{Te}$. From the theoretical point of view, there is not a strong motivation to prefer the study of one isotope over the others. For this reason, different collaborations chose different nuclei and technological approaches. In this contribution we review the status of the CUPID-0 experiment, that is searching for the $0 v \beta \beta$ of ${ }^{82} \mathrm{Se}$ with cryogenic calorimeters.

\section{The CUPID-0 Detector}

The CUPID-0 detectors exploits cryogenic calorimeters. This technology, originally proposed by Fiorini and Niinikoski [?] is characterised by an exquisite energy resolution $(<1 \%)$, a high efficiency for the containment of the two electrons emitted by $0 v \beta \beta(>80 \%)$ and, as recently proved by the CUORE experiment [3, 4], by the possibility of reaching source masses of hundreds of $\mathrm{kg}$. In addition, the crystals to be operated as calorimeters can be grown starting from different nuclei of interest for $0 v \beta \beta$, allowing an independent cross-check in case of discovery.

The main limit of cryogenic calorimeters resides in the background in the region of interest, of the order of $10^{-2}$ counts $/ \mathrm{keV} / \mathrm{kg} / \mathrm{yr}$. Such background stems mainly from $\alpha$ particles emitted by radio-active contaminants located in the materials constituting the detector [5]. A technique to suppress such background consists in coupling each calorimeter to a light detector, in order to exploit the light emission to discriminate the electrons produced (possibly) by $0 v \beta \beta$ decays from the dominant $\alpha$ background. The intense R\&D activity of the last years $[6,7,8]$ resulted in a mature and viable technology for a next-generation project: CUPID [9].

The CUPID-0 experiment, described in this contribution, is the first medium-scale demonstrator of CUPID.

The $0 v \beta \beta$ emitter chosen by the CUPID- 0 collaboration is ${ }^{82} \mathrm{Se}$. This isotope is characterised by high Q-value (2997.9 $\pm 0.3 \mathrm{keV}[10])$ and a rather long half-life for the $2 v \beta \beta$ mode $\left(\mathrm{T}_{1 / 2}^{2 v}=[9.39\right.$ \pm 0.17 (stat) \pm 0.58 (syst) $] \times 10^{19} \mathrm{yr}[11]$ ), that allows to prevent background events in the region of interest due to pile-up of $2 v \beta \beta$ decays. We increased the isotopic abundance of natural Se up to $96.3 \%$ via isotopic enrichment, and used the obtained material to synthesise the ZnSe powder and grow 24 cylindrical crystals, doped with $\mathrm{ZnSe}(\mathrm{Al})$ to enhance the light output [12]. The 24 crystals feature a total mass of $9.65 \mathrm{~kg}$, but two of them were later discarded because of their poor bolometric features, resulting in a final mass of $8.74 \mathrm{~kg}\left(3.41 \times 10^{25}\right.$ nuclei of $\left.{ }^{82} \mathrm{Se}\right)$. The detector comprises also two natural crystals, not used for the analysis of the $0 v \beta \beta$ decay.

The scintillation light emitted by the ZnSe detectors (a few \% of the total energy converted into heat) is detected using cryogenic light detectors (LD). These devices consist in high purity germanium disks operated as cryogenic calorimeters [13]. 
The ZnSe crystals, surrounded by a VIKUITI multi-layer reflecting foil produced by $3 \mathrm{M}$, and coupled to their LD, are arranged in 5 towers using PTFE elements and high purity copper frames. Both the $\mathrm{ZnSe}$ and the $\mathrm{LD}$ are equipped with Si Joule resistors injecting a periodical reference pulse to correct thermal drifts.

The description of the detector set-up, the cryogenic facility, the electronics and data-acquisition system can be found in Ref. [14].

The cool down of the CUPID-0 detector begun in January 2017 and the commissioning (comprising a first long background measurement) was completed in May 2017. We acquired physics runs for an exposure of $3.44 \mathrm{~kg} \cdot \mathrm{yr}$, published in Ref. [15], and later other $2.02 \mathrm{~kg} \cdot \mathrm{yr}$, followed by ${ }^{a}{ }^{56} \mathrm{Co}$ calibration of about 1 month. The results obtained with the full statistics are presented in this contribution. The CUPID-0 experiment will continue taking data to reach a total exposure of $10 \mathrm{~kg} \cdot \mathrm{yr}$ to improve the background model.

\section{Results}

The region of interest for CUPID-0 is a $\sim 400 \mathrm{keV}$ region centred around the Q-value of ${ }^{82} \mathrm{Se}$ : 2800-3200 keV.

After applying a basic pulses selection in order to discard non-particle like events, and after requiring that a single crystal triggered, we obtain a rate of $(3.2 \pm 0.4) \times 10^{-2}$ counts $/ \mathrm{keV} / \mathrm{kg} / \mathrm{yr}$ in the analysis window. The efficiency of this data selection is computed using the most prominent peak in the physics spectrum, produced by the ${ }^{65} \mathrm{Zn}$ decay, and results $(95 \pm 2) \%$.

We then study the most sensitive shape parameter of the filtered light pulses, defined as $\frac{1}{A \omega_{R}} \sqrt{\sum_{i-i_{M}}^{i_{M}+\omega_{R}}\left(y_{i}-A s_{i}\right)^{2}}$, where $y_{i}$ is the pulse, A and $i_{M}$ its amplitude and maximum position, $s_{i}$ the ideal signal pulse scaled to unitary amplitude and aligned to $y_{i}, \omega_{R}$ the right width at half maximum of $s_{i}$. As described in Ref. [16], we optimise this shape parameter in order to have $100 \%$ efficiency on the signal while reducing the $\alpha$ background. The signal efficiency is computed on a pure signal-like sample using electrons produced by $\mu$ interactions, that result in high multiplicity events (more than 5 crystals trigger simultaneously). The counting rate in the analysis window after the selection on the light pulses decreases to $(1.3 \pm 0.2) \times 10^{-2}$ counts $/ \mathrm{keV} / \mathrm{kg} / \mathrm{yr}$, proving that the dual read-out of light and heat effectively suppress the background in the RoI.

We further reduce the background by identifying the events produced by ${ }^{208} \mathrm{Tl}$ decays in the region of interest. To tag these events, we exploit the short half-life of ${ }^{208} \mathrm{Tl}$ (about 3 minutes), and the fact that a ${ }^{208} \mathrm{Tl}$ decay is preceded by an $\alpha$ decay of its mother, ${ }^{212} \mathrm{Bi}$. Whenever we tag the $\alpha$ particle emitted by ${ }^{212} \mathrm{Bi}$, we open a $\sim 9$ minutes time-veto in order to reject possible ${ }^{208} \mathrm{Tl}$ events.

The $\alpha$ particles emitted by ${ }^{212} \mathrm{Bi}$ are expected to produce a peak at $6.21 \mathrm{MeV}$ (this value includes also the nuclear recoil). Nevertheless, if ${ }^{212} \mathrm{Bi}$ is located on the surface of the crystal, we could lose a variable fraction of energy, and observe an $\alpha$ event at much lower energy. For this reason, we decided to exploit the excellent discrimination capability of CUPID- 0 to tag $\alpha$ particles down to $\sim 2 \mathrm{MeV}$, and open the time veto whenever an $\alpha$ particle with energy between $2 \mathrm{MeV}$ and the $\mathrm{Q}$-value of ${ }^{212} \mathrm{Bi}$ interacts. After applying the time-veto, the event rate in the analysis window drops to $3.2_{-1.1}^{+1.3} \times 10^{-3}$ counts $/ \mathrm{keV} / \mathrm{kg} / \mathrm{yr}$. The final efficiency for the search of $0 v \beta \beta$ decay (containment of the $0 v \beta \beta$ electrons + trigger + energy reconstruction + data selection $+\alpha$ rejection and time-veto) amounts to $(75 \pm 2) \%$. 
In conclusion, CUPID-0 set the first important milestone for a next generation experiment based on cryogenic calorimeters, proving that the CUORE background $\left(\sim 10^{-2}\right.$ counts $\left./ \mathrm{keV} / \mathrm{kg} / \mathrm{yr}\right)$ can be reduced to $3.2_{1.1}^{1.3} \times 10^{-3}$ counts $/ \mathrm{keV} / \mathrm{kg} / \mathrm{yr}$ by exploiting the simultaneous read-out of light and heat. Thanks to the low background and high efficiency, it was possible to set the most competitive lower limit on the half-life of ${ }^{82} \mathrm{Se}: \mathrm{T}_{1 / 2}^{0 v}>4.0 \times 10^{24} \mathrm{yr}$ (90\% C.I.).

\section{References}

[1] W. H. Furry, Phys. Rev. 56, 1184 (1939).

[2] S. Dell'Oro, S. Marcocci, M. Viel, F. Vissani, Adv. High Energy Phys.2016, 2162659 (2016). bibitemFiorini:1983yj E. Fiorini, T.O. Niinikoski, Nuclear Instruments and Methods in Physics Research A 224, 83 (1984).

[3] D.R. Artusa, et al., Adv. High Energy Phys. 2015, 879871 (2015).

[4] C. Alduino et al., [CUORE Collaboration], Phys. Rev. Lett. 120(13), 132501 (2018).

[5] C. Alduino et al., [CUORE Collaboration], Eur. Phys. J. C 77 (2017) no.8, 543.

[6] J.W. Beeman, et al., JINST 8, P05021 (2013).

[7] L. Cardani, et al., JINST 8, P10002 (2013).

[8] D. R. Artusa et al., Eur. Phys. J. C 76 no.7, 364 (2016).

[9] D. Artusa, et al., Eur. Phys. J. C 74(10), 3096 (2014).

[10] D.L. Lincoln, et al., Phys.Rev.Lett. 110, 012501 (2013).

[11] R. Arnold et al., arXiv:1806.05553 (2018)

[12] I. Dafinei et al., J. Cryst. Growth 475158 (2017).

[13] J.W. Beeman, et al., JINST 8, P07021 (2013).

[14] O. Azzolini et al., Eur.Phys.J. C 78 no.5, 428 (2018).

[15] O. Azzolini et al., Phys. Rev. Lett. 120232502 (2018).

[16] O. Azzolini et al., Eur.Phys.J. C 78 no.9, 734 (2018). 